\title{
Administration of JTE013 abrogates experimental asthma by regulating proinflammatory cytokine production from bronchial epithelial cells
}

Tomomi Terashita, Kazuyuki Kobayashi*, Tatsuya Nagano, Yoshitaka Kawa, Daisuke Tamura, Kyosuke Nakata, Masatsugu Yamamoto, Motoko Tachihara, Hiroshi Kamiryo and Yoshihiro Nishimura

\begin{abstract}
Background: Sphingosine-1-phosphate (S1P) is a bioactive phospholipid that acts as a signal transducer by binding to S1P receptors (S1PR) 1 to 5. The S1P/S1PRs pathway has been associated with remodeling and allergic inflammation in asthma, but the expression pattern of S1PR and its effects on non-immune cells have not been completely clarified. The aim of this study was to examine the contribution of the signaling of S1P and S1PRs expressed in airway epithelial cells (ECs) to asthma responses in mice.

Methods: Bronchial asthma was experimentally induced in BALB/C mice by ovalbumin (OVA) sensitization followed by an OVA inhalation challenge. The effects of S1PR antagonists on the development of asthma were analyzed $24 \mathrm{~h}$ after the OVA challenge.

Results: Immunohistological analysis revealed S1PR1-3 expression on mouse airway ECs. Quantitative real-time polymerase chain reaction demonstrated that S1P greatly stimulated the induction of CCL3 and TIMP2 mRNA in human airway ECs, i.e., BEAS-2B cells, in a dose-dependent manner. Pretreatment with the S1PR2 antagonist JTE013 inhibited the CCL3 gene expression in BEAS-2B cells. Immunohistological analysis also showed that the expression level of CCL3 was attenuated by JTE013 in asthmatic mice. Furthermore, JTE013 as well as anti-CCL3 antibody attenuated allergic responses. Intratracheal administration of JTE013 also attenuated eosinophilic reactions in bronchoalveolar lavage fluids. S1P induced transcription factor NFKB activation, while JTE013 greatly reduced the NFKB activation.
\end{abstract}

Conclusions: JTE013 attenuated allergic airway reactions by regulating CCL3 production from bronchial ECs. The intratracheal administration of JTE013 may be a promising therapeutic strategy for bronchial asthma.

Keywords: Sphingosine-1-phosphate, epithelial cells, BEAS-2B, JTE013, CCL3

\section{Background}

Sphingosine-1-phosphate (S1P) is a bioactive phospholipid metabolite [1]. It is produced by most kinds of cells through phosphorylation by sphingosine kinases (SPHK1 and SPHK2), and it works as a signal transducer of intracellular and extracellular cell homeostasis and functions, such as cell differentiation, inflammation, and apoptosis [2]. Outside of the cell, S1P binds to five G-

\footnotetext{
* Correspondence: kkoba@med.kobe-u.ac.jp

Division of Respiratory Medicine, Department of Internal Medicine, Kobe University Graduate School of Medicine, 7-5-1 Kusunoki-cho, Chuo-ku, Kobe 650-0017, Japan
}

protein-coupled receptors, i.e., S1P receptors (S1PR) 1 to 5 , as a ligand. These receptors are expressed mainly by inflammatory cells, and they play an important role in immunity. The individual expression pattern of S1PRs on each cell creates diverse immunological responses [3]. However, the expression pattern of S1PR and its effects on non-immune cells remain unclear. Therefore, we performed a functional analysis of sphingolipids foon the roles of SIP and SIPRs on non-immune ce reported that TGF- $\beta$ increases SPHK1, and consequently S1P, which transactivates S1PR2 and S1PR3 on fibroblasts, and promotes their transformation 
to myofibroblasts through Rho kinase $[4,5]$. We further reported that SPHK1 mediates the transformation of airway epithelial cells (ECs) to goblet cells [6]. We also clarified that the intratracheal administration of N,Ndimethylsphingosine (DMS), a S1P synthesis inhibitor, restrained allergic inflammation and remodeling [7]. Thus, S1P and S1PRs play crucial roles in remodeling and allergic inflammation in asthma.

Bronchial asthma is a cytokine-based inflammatory dysfunction that involves airway ECs, endothelial cells, and inflammatory cells, such as Th2-type lymphocytes, dendritic cells, and mast cells [8]. Healthy airway ECs, which provide a physical barrier, can keep mast cells in a quiescent state and inhibit mast cell degranulation [3]. However, damage to airway ECs due to inflammation initiates innate immune responses, and stimulates Th2 responses by directly attracting and activating immune cells, such as Th2 cells, eosinophils, and mast cells [9-13]. Clarification of the functions of airway ECs and efforts to minimize airway EC damage are important for asthma treatment.

In this study, we investigated the expression of S1PRs on airway ECs and the function of airway ECs in the signaling pathway of the S1P/S1PRs axis by using an experimental bronchial asthma mouse model. We used human bronchial ECs, i.e., BEAS-2B cells, stimulated with S1P for identifying related cytokines to investigate the signaling pathway, and to evaluate the potential of S1PR inhibitors as a remedy for bronchial asthma.

\section{Methods}

\section{Reagents}

S1P (PeproTech, Rocky Hill, NJ) was used. A S1PR2 antagonist (also known as JTE013; Cayman, Ann Arbor, $\mathrm{MI}$ ), and a S1PR3 antagonist (also known as VPC23019; Calbiochem, Darmstadt, Germany) were purchased. The antibodies used were anti-S1PR1-5 (Cayman) and anti-CC chemokine ligand (CCL) 3 (R\&D Systems, Minneapolis, MN).

\section{Animals}

Female BALB/c mice were purchased from SLC Japan (Shizuoka, Japan). All mice were raised in sterile cages, and were used at 7 to 10 weeks of age. Our studies were approved by the Institutional Animal Care and Use Committee (Permit Numbers: P130610-R1, and P150804), and carried out in accordance with the Kobe University Animal Experimentation Regulations.

\section{Experimental mouse models}

The experimental bronchial asthma model mice were prepared by sensitization with intraperitoneal (i.p.) injections of $10 \mu \mathrm{g}$ of ovalbumin (OVA; Sigma-Aldrich, St. Louis, MO) and $2 \mathrm{mg}$ of aluminum hydroxide (Sigma-
Aldrich) in $0.5 \mathrm{ml}$ of sterile phosphate-buffered saline (PBS) on days 0 and 7. Then, on days 21 and 22, the mice were exposed to aerosolized sterile PBS with or without $1.0 \%$ OVA for 30 min with ultrasonic nebulizer, Omron NE-U07 (OMRON, Kyoto, Japan) (the aerosolized particle size ranges $1-8 \mu \mathrm{m})$. Mice were sacrificed on day 23 for all mice experimentations. To prepare JTE013-treated or anti-CCL3 antibody-treated mice, mice were given JTE013 $(4 \mathrm{mg} / \mathrm{kg})$ or anti-CCL3 antibody $(0.15 \mathrm{mg} / \mathrm{kg})$ via i.p. injection before OVA sensitization on days 21 and 22. In the JTE013 intratracheal administration experiment, mice were intratracheally instilled with $10 \mu \mathrm{g}$ of JTE013 or PBS.

\section{Analysis of bronchoalveolar lavage fluids}

Bronchoalveolar lavage (BAL) fluids were collected by lavaging lungs three times with $800 \mu \mathrm{l}$ of PBS, and samples were stored at $-80{ }^{\circ} \mathrm{C}$ after centrifugation. The concentrations of interleukin (IL)-4, IL-5, IL-13, and IFN- $\gamma$ in the BAL fluids were measured using the Mouse ELISA Kit (R\&D Systems) according to the manufacturer's instructions.

\section{Histology}

Lungs were harvested to prepare paraffin-embedded sections for histological analysis. PBS was perfused through the lungs of mice under deep anesthesia, and the lungs were then fixed by intratracheal instillation of phosphatebuffered paraformaldehyde (Wako, Osaka, Japan). Airway EC areas were evaluated in five randomly selected sections, and each section was subjected to hematoxylin and eosin staining and immunostaining, as previously described $[14,15]$.

\section{Cell culture and stimulation}

BEAS-2B cells (ATCC, Manassas, VA) were incubated with the BEGM Bullet Kit (Lonza, Walkersville, MD) under a humidified atmosphere containing $5 \% \mathrm{CO}_{2}$ at $37^{\circ} \mathrm{C}$. To analyze the secretion of cytokines, the cells were stimulated with or without S1P $(100 \mathrm{nM}$ or $1 \mu \mathrm{M})$ for $3 \mathrm{~h}$. In other experiments, cells were treated with $1 \mu \mathrm{M}$ S1P and $10 \mu \mathrm{M}$ JTE013, $10 \mu \mathrm{M}$ VPC23019, $50 \mu \mathrm{M}$ S3I-201 (STAT3 inhibitor, Cosmo Bio Co, Tokyo, Japan), $2.9 \mu \mathrm{M}$ IKK inhibitor III (Calbiochem), or dimethyl sulfoxide (DMSO).

Quantitative real-time polymerase chain reaction analysis Total cellular RNA was prepared and quantitative realtime polymerase chain reaction (qRT-PCR) was performed as previously described [16]. Relative human mRNA levels were calculated with the $\Delta \Delta \mathrm{Ct}$ method using glyceraldehyde-3-phosphate dehydrogenase mRNA as an internal control. The sequences of the sense and antisense primers are listed in Additional file 1: Table S1. 


\section{Western blotting analysis}

The preparation of cell lysates, sodium dodecyl sulfatepolyacrylamide gel electrophoresis, and immunoblotting were performed as previously described [17]. Primary antibodies against NFKB (Santa Cruz Biotechnology, Dallas, TX) and $\beta$-actin (Cell Signaling, Danvers, MA), and secondary antibodies (Cell Signaling) were used.

\section{Statistical analysis}

Data are shown as the mean \pm standard error of the mean (SEM). Statistical analyses were performed using the Student's unpaired $t$-test to compare two groups. The significances of all statistical tests are provided in the figures. A $p$-value of $<0.05$ was considered to be statistically significant.

\section{Results}

S1PR1-3 is expressed on airway epithelial cells in vivo Previous studies suggested the existence of S1PRs on airway ECs, because the administration of DMS to ECs mitigated allergic reactions [6, 7]. Thus, we investigated the localization of S1PRs on ECs by immunostaining with S1PRs antibodies. As shown in Fig. 1, S1PR1, S1PR2, and S1PR3 were expressed in airway ECs, including bronchial ECs and alveolar ECs. These results suggest that the S1P/S1PR1-3 axis in airway ECs has a possible role in airway inflammation.

\section{S1P stimulation of airway ECs evidently induces CCL3 and TIMP2 gene expression in vitro}

Next, we analyzed the function of the S1P/S1PR1-3 axis in cytokine secretion by using BEAS-2B human airway ECs. Comparison by qRT-PCR of the cytokine mRNA levels of S1P- or DMSO-treated BEAS-2B cells indicated that stimulation with $\mathrm{S} 1 \mathrm{P}$ promoted the expression of CCL3 and TIMP2 (Fig. 2a).

\section{CCL3 and TIMP2 are S1P-dependent in vitro and in vivo}

We further analyzed the dose-dependent CCL3 and TIMP2 gene expression in BEAS-2B cells after stimulation with S1P. As shown in Fig. 2b, CCL3 and TIMP2 gene expression in BEAS-2B cells increased in proportion to the S1P concentration, and they were attenuated by JTE013, a S1PR2 antagonist (Fig. 2c). In contrast, neither was attenuated by VPC23019, a S1PR1 and S1PR3 antagonist (Fig. 2c). Immunohistological analysis also showed that CCL3 and S1PR2 were co-expressed on the airway ECs in the experimental asthma mouse model, and the expression level of CCL3 was attenuated by JTE013, although the expression level of S1PR2 was not attenuated by JTE013 because JTE013 only inhibits S1P binding to S1PR2 (Fig. 3a). further analyzed the effect of CCL3 on airway allergic response using the experimental asthma mouse model. As shown in Fig. 3b, airway

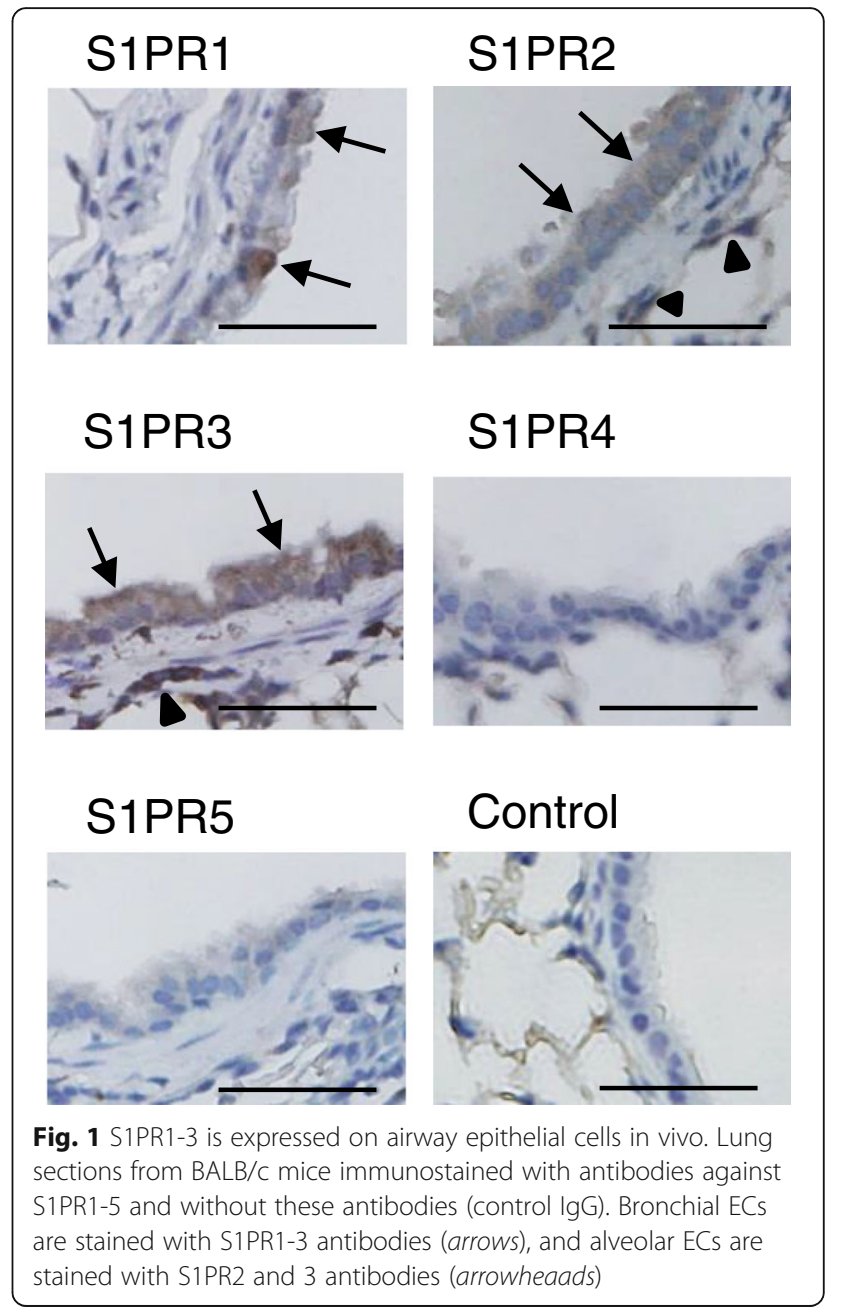

eosinophilia and the levels of IL-4, IL-5, and IL-13 were attenuated by the anti-CCL3 antibody. These results suggest that S1P induced the secretion of CCL3, which has a crucial role in bronchial asthma through the S1P/ S1PR2 axis in airway ECs.

\section{JTE013 decreases allergic responses}

To investigate the effects of the S1P/S1PR2 axis on bronchial asthma, we employed an experimental asthma mouse model and evaluated the therapeutic effects of JTE013. As expected, OVA-treated mice showed a marked increase in the number of eosinophils in the BAL fluids (Fig. 4a). In addition, the levels of IL-4, IL-5, and IL-13 were increased in the BAL fluids from OVAtreated mice (Fig. 4b). In contrast, JTE013 administration resulted in a significant decrease in Th2 inflammatory reactions, although there was no statistical difference in the levels of IL-4 (Fig. 4a and b). Consistent with the BAL fluids analysis, histological analysis demonstrated a marked decrease in inflammatory cell infiltration around 


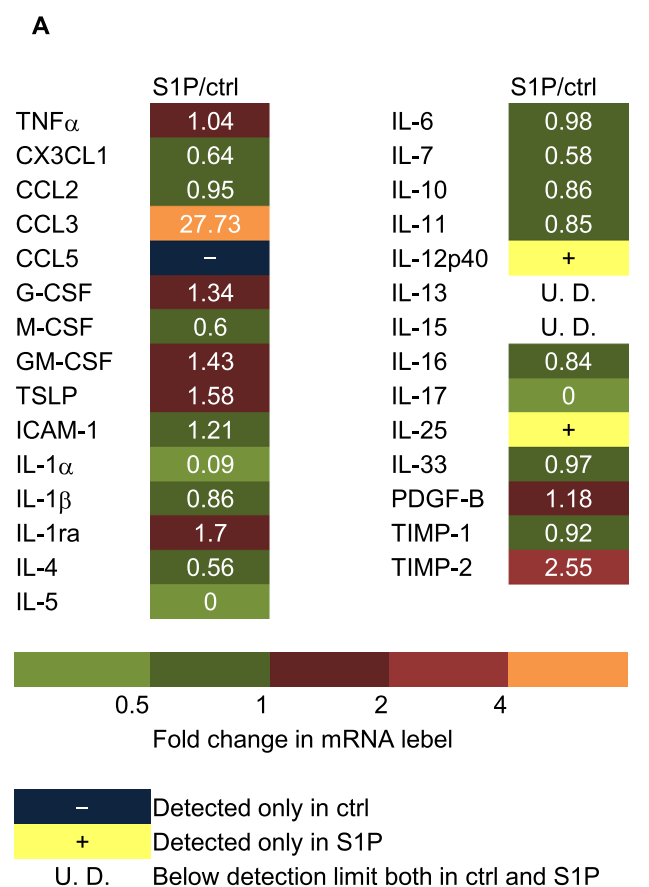

\section{B}

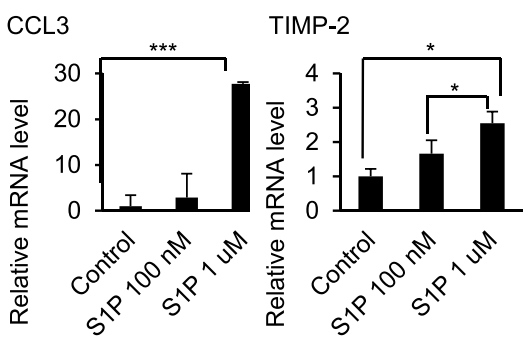

C

CCL3

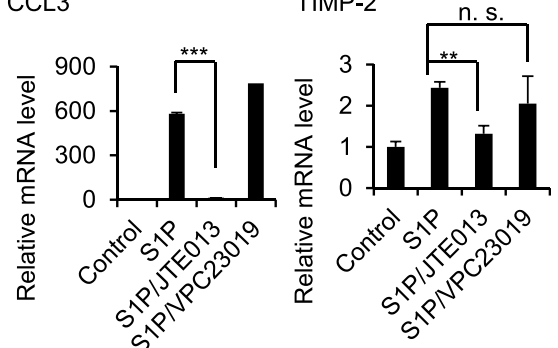

Fig. 2 S1P stimulation of airway ECs induces CCL3 and TIMP2 gene expression, and CCL3 and TIMP2 are S1P-dependent in vitro. BEAS-2B cells were cultured with or without S1P (100 nM). The mRNA expression of 29 cytokines was analyzed by quantitative real-time RT-PCR. Data represent the ratio between the relative mRNA level of S1P-treated cells and that of S1P-untreated cells (a). BEAS-2B cells were treated with S1P (100 nM or $1 \mu \mathrm{M})(\mathbf{b})$, S1P $(1 \mu \mathrm{M})$ and JTE013 $(10 \mu \mathrm{M})$, or S1P $(1 \mu \mathrm{M})$ and VPC23019 $(10 \mu \mathrm{M})(\mathbf{c})$ for 3 h, and CCL3 and TIMP2 gene expression was analyzed by quantitative real-time RT-PCR
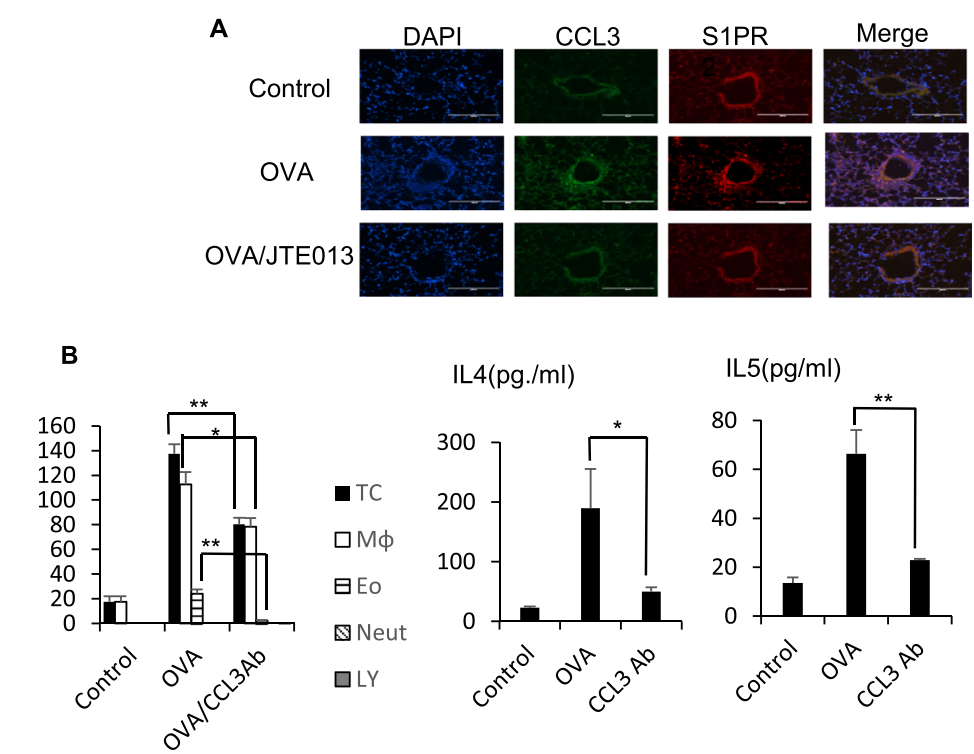

IL13(pg/ml)

Fig. 3 CCL3 and TIMP2 are S1P-dependent in vivo. Immunofluorescent microscopic images show OVA-treated lung sections stained with FITCconjugated anti-CCL3 (green), and Alexa 594-conjugated anti-S1PR2 (red) antibodies. Co-localization of CCL3 and S1PR2 is seen in yellow (a). BAL Fluids were obtained from BALB/c mice treated with vehicle, OVA, or OVA/mCCL3 antibody (3 $\mu \mathrm{g} / \mathrm{cavity}$ ), and examined by Diff-Quick staining. The total cell counts and cell differentials in the BAL fluids are shown, and the concentrations of IL-4, IL-5, and IL-13 in the BAL fluids were measured by using ELISA kits (b). Data are expressed as the mean \pm standard error (SE) of at least three independent experiments. ${ }^{*} p<0.05,{ }^{* *} p<0.01,{ }^{* * *} p<0.001$ 


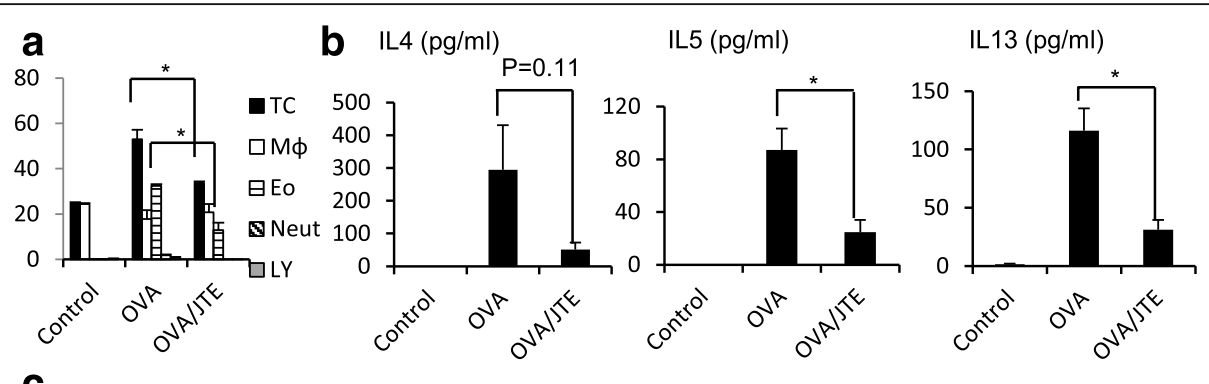

C PBS

OVA

OVAVJTE013

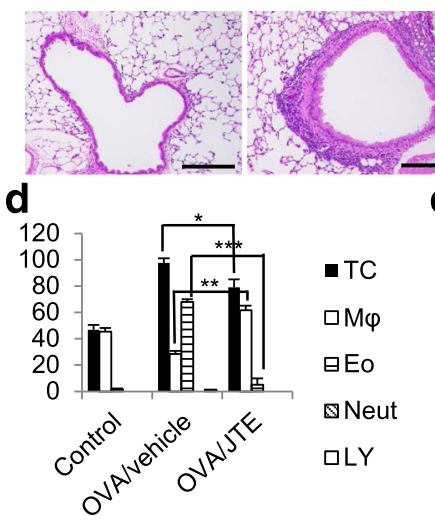

e

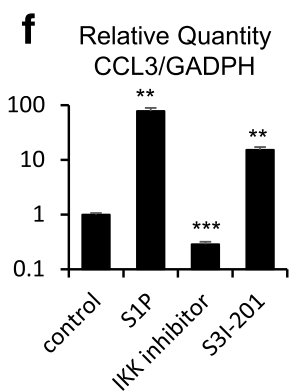

Fig. 4 JTE013 decreases allergic responses. BAL fluids were obtained after OVA or vehicle exposure, and examined by Diff-Quick staining. Total cell counts and cell differentials in the BAL fluids are shown (a), and the concentrations of IL-4, IL-5, IL-13, and IFN- $\gamma$ in the BAL fluids were measured by using ELISA kits $(n=5)(\mathbf{b})$. Lung sections from BALB/c mice treated with vehicle, OVA, or OVA/JTE013 (4 mg/kg) were subjected to hematoxylin and eosin staining (c). BAL fluids were obtained after OVA or vehicle exposure with intratracheal administration of vehicle or JTE013 (10 $\mu$ g/trachea), and the total cell counts and cell differentials in the BAL fluids were examined by Diff-Quick staining $(n=3$ to 5$)(\mathbf{d})$. BEAS-2B cells were treated with vehicle, S1P $(1 \mu \mathrm{M})$, or S1P/JTE013 $(1 \mu \mathrm{M})$ for $4 \mathrm{~h}$. Phosphorylation of p65 was evaluated by western blot analysis. Representative images from three independent experiments are shown (e). BEAS-2B cells were treated with vehicle, S1P (1 MM), IKK inhibitor, or S3I-201. Each CCL3 gene expression was analyzed by quantitative real-time RT-PCR, representing the relative $\mathrm{mRNA}$ level of CCL3 (f). Data are expressed as the mean \pm SE of at least three independent experiments. ${ }^{*} p<0.05,{ }^{* *} p<0.01,{ }^{* * *} p<0.001$

the bronchiole in OVA/JTE013-treated mice when compared to OVA-treated mice (Fig. 4c).

Moreover, intratracheal administration of JTE013 also resulted in a marked decrease in eosinophilic reactions in the BAL fluids (Fig. 4d). This suggests that intratracheal treatment can inhibit allergic bronchial inflammation as well as i.p. treatment.

\section{JTE013 inhibits CCL3 gene expression by attenuating NFKB and STAT3 activation in vitro}

Next, we analyzed the signaling pathways downstream of S1PR2, and investigated the activation of transcription factors, NFkB and STAT3. Previous study reported that S1PR2 can activate the transcription factor, STAT3 in mice lung [18], which regulates CCL3 expression in macrophage [19], and the transcription factor $\mathrm{NF}_{\mathrm{K} B}$ also induces CCL3 synthesis in nucleus pulposus cells [20]. In this study, using BEAS-2B cells, we first assessed the activity of NFKB downstream of the S1P/S1PR2 signaling pathway, and next analyzed the CCL3 expression downstream of NFKB and STAT3 activation. The results are shown in
Fig. $4 \mathrm{e}$ and $\mathrm{f}$. The expression of NFkB increased after stimulation with S1P, while it decreased with JTE013 (Fig. 4e), and CCL3 gene expression in BEAS-2B cells increased with the S1P concentration, while it was attenuated by IKK inhibitor, and a STAT3 inhibitor, S3I-201. Taken together, these results suggest that JTE013 inhibits CCL3 expression through NFKB and STAT3 transcription.

\section{Discussion}

The aim of this study was to elucidate the role of S1P in bronchial asthma by focusing on airway ECs. Pathological analysis showed that S1PR1-3 are expressed on airway ECs, and airway ECs that expressed S1PR2 also expressed CCL3 at a high level in response to the OVA inhalation challenge. Our previous research showed the inflammation induced by OVA exposure decreases in time-dependent manner [17]. Therefore, we adopted our time schedule as appropriate duration time.

In vitro analysis of cytokine expression in human bronchial ECs also showed that S1P led to the transcriptional activation of the CCL3 gene through $\mathrm{NFKB}$ and 
STAT3 activation. Previous studies reported that S1PR2 is coupled to $\mathrm{G} \alpha \mathrm{I}, \mathrm{G} \alpha 12 / 13$, and $\mathrm{G} \alpha \mathrm{Q}$, which activates phospholipase C (PLC) [21, 22]. PLC plays a role in the regulation of intracellular signaling pathways through calcium release and protein kinase $\mathrm{C}$ activation [22]. PLC also plays a role in proinflammatory gene expression through the activation of NFkB [23]. These results suggest that the S1P/S1PR2 axis has a possible role in cytokine expression via the activation of NFKB downstream of PLC. Furthermore, STAT3 is directly activated by S1PR2 in a Rho-dependent manner [18, 24], which is dependent on the S1PR2-coupled G protein Go12/13 [21].

We used human bronchial ECs instead of mouse bronchial ECs as previous literatures [25-27], because previous reports showed that approximately $99 \%$ of mouse genes have a homologue in the human genome [28], and bioinformatic analysis and human curation of 60,770 full-length mouse cDNA clones showed similarity (70-85\% identity) to known human-disease genes [29].

CCL3 expression is up-regulated by NFkB and STAT3. Consistent with our results, Jianru $\mathrm{W}$ et al. also demonstrated that NFKB introduces CCL3 synthesis, by binding to the proximal promotor of CCL3 major nuclear proteins in nucleus pulposus cells [20]. Furthermore, Zhang $\mathrm{C}$ et al. also reported that STAT3 is a key regulator of CCL3 expression in macrophages [19]. Taken together, we infer that the S1P/S1PR2 axis activates STAT3 and NFKB to form a complex with CCL3 nuclear protein binding sites, which in

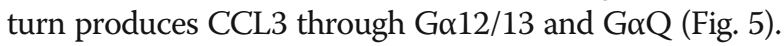

CCL3 is a member of the C-C chemokine family, and is a chemoattractant for eosinophils, lymphocytes,

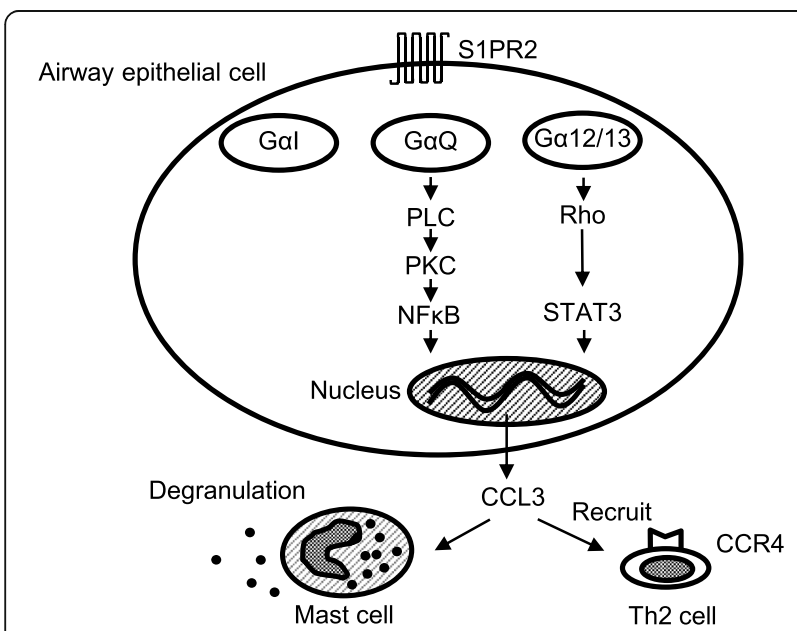

Fig. 5 Simplified scheme of the S1PR2 signaling events in airway epithelial cells. On airway epithelial cells, S1P binds to S1PR2, which is coupled to Gal, Ga12/13, and GaQ. GaQ in turn activates PLC, activating PKC. PKC phosphorylates NFKB, which binds to the proximal promotor of CCL3 major nuclear proteins. In addition, another S1PR2-coupled G protein, Ga12/13, also up-regulates CCL3 expression through STAT3 activation in a Rho-dependent manner leukocytes, and monocytes. Previous studies reported that an increased level of CCL3 was observed in BAL fluids from patients with asthma [30], and that CCL3 evoked local $\mathrm{T}$ cell recruitment and induced skin reactions in patients with atopic dermatitis [31]. Furthermore, Elena et al. demonstrated significantly higher levels of CCL3 in BAL fluids from patients with corticosteroid-resistant asthma [32].

CCL3 is also a ligand for CCR1 and CCR4. CCR1 is expressed in mast cells and basophils, and the CCL3/ CCR1 axis is important for the activation of mast cells [33]. A previous study reported that CCL3 acted as a costimulator for FceRI-mediated degranulation in conjunctival mast cells in CCL3-deficient mice [34]. Moreover, CCL3 synergistically enhanced FceRI-mediated degranulation and the production of CCL2, which is a chemoattractant for $\mathrm{T}$ cells and eosinophils in bone marrowderived murine mast cells and the rat basophilic leukemia $2 \mathrm{H} 3$ cell line [33, 35-39]. Taken together, there is crosstalk between CCR1-mediated and FceRI-mediated signaling cascades in mast cells, which plays an important role in allergic disorders as FceRI cross-linking is a key event of activation. On the other hand, CCR4 mediates allergypromoting Th2 cell recruitment [23]. A recent publication by Oskeritzian et al. demonstrated that S1P neutralization or a S1PR2 antagonist strongly attenuated early T-cell recruitment to lung perivascular lesions and T-cell chemokine production, including CCL3, in murine mast celldependent acute airway allergic responses [23], suggesting that the S1P/S1PR2 axis regulates early T-cell recruitment via chemokine production. In our study, immunohistochemical analysis showed that CCL3 was abundantly produced by airway ECs. Therefore, CCL3 on airway ECs may be a promising target for the treatment of airway allergic inflammation, which is mediated by T-cell recruitment. In fact, our study showed that CCL3 neutralization actually reduced Th2-type allergic responses.

The importance of S1P in an experimental asthma mouse model is well established [4-7]. Past reports have suggested that the S1P/S1PR2 axis also plays an important role in antigen-induced mast cell degranulation [40], myofibroblast contraction [41], and vasoconstriction [42]. Our study first verified the contribution of the signaling of the S1P/S1PR2 axis in airway ECs. However, the intraperitoneal administration of S1PR2 antagonist to investigate the in vivo reaction of airway ECs can also induce a systemic reaction, as described above. Although we examined the intratracheal administration of a S1PR2 antagonist, the possibility cannot be excluded that the method could also induce systemic reaction through resident mast cells around airway ECs. In order to determine the effects of the S1PR2 antagonist on airway ECs in vivo, an appropriate experimental mouse model, such as S1PR2-deficient mice [43], should be employed. 
Our in vitro study using primary cultures demonstrated that the S1P/S1PR2 axis promotes airway inflammation. Intriguingly, Yapeng et al. showed that S1PR2 induced the extrusion of apoptotic kidney ECs to maintain an intact barrier [44]. In addition, Kono et al. showed that S1PR2knockout mice had defective epithelial barrier function in the cochlea [45]. These results suggest that inhibition of the S1P/S1PR2 pathway disturbs the barrier function of ECs, and may aggravate asthmatic reactions. We have two hypotheses to account for these contradictory findings. One hypothesis is that the nature of airway ECs is different from those of other ECs. The other hypothesis is that the quantity of JTE013 used was enough to restrain inflammatory responses, but not enough to restrain the extrusion of airway ECs. Further studies investigating the effects of the S1PR2 antagonist at different intratracheal dosage levels on asthma would be of great interest.

In addition, we investigated the allergic reaction by intratrachial administration of aerosolized OVA and JTE013 to show the potential of intratrachial administration of JTE013 as the remedy of bronchial asthma. However, Kannan et al. have revealed that the deposition of particle substance to airway and lung depends on many factors including the property of the particle, anatomical geometry of the airway, and respiratory pattern [46, 47]. And it may be possible to obtain more analysis from the computational Euler-Langrangian particle delivery approach of Kannan et al. [47]. In this point, there are some limitations to interpret our in vivo data. Though extrapolating from our studies with intratrachial administration in mouse model to the allergic reaction in humans requires cautious interpretation, our data can provide significant promise to JTE013 as the remedy because previous study showed that asthma mouse model can be used to investigate the main point of our pathogenesis, allergic reaction through the cytokine production [48] and previous report showed the intratrachial administration of drugs such as budesonide in mice has similar efficacy in humans [49]. Therefore, I think our results can lead to the development of worthwhile therapeutic interventions.

\section{Conclusion}

This study demonstrated that the S1PR2 antagonist JTE013 inhibits asthmatic allergic reactions by suppressing the S1PR2-mediated NFKB activation and CCL3 production in the bronchial epthelium, and that the intratracheal administration of JTE013 may be a promising remedy for bronchial asthma.

\section{Additional file}

Additional file 1: Table S1. The primers used for qRT-PCR. (DOCX $30 \mathrm{~kb}$ )

\section{Abbreviations}

BAL: Bronchoalveolar lavage; CCL: CC chemokine ligand; DMS: N,Ndimethylsphingosine; DMSO: Dimethyl sulfoxide; ECs: Epithelial cells; i.p.: Intraperitoneal; IL: Interleukin; OVA: Ovalbumin; PBS: Phosphate buffered saline; PLC: Phospholipase C; qRT-PCR: Quantitative real-time polymerase chain reaction; S1P: Sphingosine-1-phosphate; S1PR: Sphingosine-1phosphate receptor; SEM: Standard error of the mean; SPHK: Sphingosine kinase

\section{Acknowledgements}

We thank Mr. Kei Kunimasa for his technical support.

\section{Funding}

This work was supported by JSPS KAKENHI 24591131 and Research Grants from MSD and AstraZeneca K.K. to Yoshihiro Nishimura, and JSPS KAKENHI 23591119 to Kazuyuki Kobayashi.

\section{Availability of data and materials}

All data generated or analysed during this study are included in this article and its supplementary information files.

\section{Authors' contributions}

$\Pi T, K K$, and TN analyzed the data and $T T$ drafted the manuscript. KK conceived of the study, and participated in its design. TN participated in its design and helped to draft the manuscript. YK assisted with the histological studies. DT and KN participated in the statistical analysis. MY, TM, and YN participated in the design and assited with the technical advice. ALL the authors read and approved the final manuscript.

\section{Competing interests}

The authors declare that they have no competing interests.

\section{Consent for publication}

Not applicable.

\section{Ethics approval}

All animal experiments were approved by the Institutional Animal Care and Use Committee (Permit Numbers: P130610-R1, and P150804), and carried out in accordance with the Kobe University Animal Experimentation Regulations.

Received: 3 October 2016 Accepted: 1 November 2016

Published online: 09 November 2016

\section{References}

1. Rivera J, Proia RL, Olivera A. The alliance of sphingosine-1-phosphate and its receptors in immunity. Nat Rev Immunol. 2008;8:753-63.

2. Rivera J, Olivera A. Src family kinases and lipid mediators in control of allergic inflammation. Immunol Rev. 2007;217:255-68.

3. Sanchez T, HIa T. Structual and functional characteristics of S1P receptors. J Cell Biochem. 2004;92:913-22.

4. Urata $Y$, Nishimura $Y$, Hirase T, Yokoyama M. Sphingosin 1-phosphate induces alpha-smooth muscle actin expression in lung fibroblasts via Rho-kinase. Kobe J Med Sci. 2005;51(1-2):17-21.

5. Kono Y, Nishiuma T, Nishimura Y, Kotani Y, Okada T, Nakamura S, et al. Sphingosine kinase 1 regulates differentiation of human and mouse lung fibroblasts mediated by TGF- $\beta 1$. Am J Respir Cell Mol Biol. 2007;27:395-404.

6. Kono Y, Nishiuma T, Okada T, Kobayashi K, Funada Y, Kotani Y, et al. Sphingosine kinase 1 regulates mucin production via ERK phosphorylation. Pulm Pharmacol Ther. 2010;23:36-42.

7. Nishiuma T, Nishimura Y, Okada T, Kuramoto E, Kotani $Y$, Jahangeer $\mathrm{S}$, et al. Inhalation of sphingosine kinase inhibitor attenuates airway inflammation in asthmatic mouse model. Am J Physiol Lung Cell Mol Physiol. 2008;294: L1085-93.

8. Peter JB. Immunology of asthma and chronic obstructive pulmonary disease. Nat Rev Immunol. 2008:8:183-92.

9. Holgate ST. The epithelium is central to the pathogenesis of asthma. Allergol Int. 2008;57:1-10.

10. Saenz SA, Taylor BC, Artis D. Welcome to the neighborhood: epithelial cell-derived cytokines license innate and adaptive immune responses at mucosal sites. Immunol Rev. 2008;226:172-90.

11. Schleimer RP, Kato A, Kern R, Kuperman D, Avila PC. Epithelium: at the interface of innate and adaptive immune responses. J AllergyClin Immunol. 2007;120:1279-84. 
12. Sansonetti PJ. War and peace at mucosal surfaces. Nat Rev Immunol. 2004;4:953-64.

13. Bulek K, Swaidani S, Aronica M, Li X. Epithelium: the interplay between innate and Th2 immunity. Immunol Cell Biol. 2010;88:257-68.

14. Nagano T, Yasunaga M, Goto K, Kenmotsu H, Koga Y, Kuroda J, et al. Antitumor activity of NK012 combined with cisplatin against small cell lung cancer and intestinal mucosal changes in tumor-bearing mouse after treatment. Clin Cancer Res. 2009;15(13):4348-55.

15. Kenmotsu H, Yasunaga M, Goto K, Nagano T, Kuroda J, Koga Y, et al. The antitumor activity of NK012, an SN-38-incorporating micelle, in combination with bevacizumab against lung cancer xenografts. Cancer. 2010;116(19):4597-604.

16. Nagano T, Yasunaga M, Goto K, Kenmotsu H, Koga Y, Kuroda J, et al. Synergistic antitumor activity of the SN-38-incorporating polymeric micelles NK012 with S-1 in a mouse model of non-small cell lung cancer. Int J Cancer. 2010;127(11):2699-706.

17. Kuramoto E, Nishiuma T, Kobayashi K, Yamamoto M, Kono Y, Funada Y, et al. Inhalation of urokinase-type plasminogen activator reduces airway remodeling in a murine asthma model. Am J Physiol Lung Cell Mol Physiol. 2009;296(3):L337-46.

18. Oskeritzian CA, Hait NC, Wedman P, Chumanevich A, Kolawole EM, Price MM, et al. The sphingosine-1-phosphate/sphingosine-1-phosphate receptor 2 axis regulates early airway T-cell infiltration in murine mast cell-dependent acute allergic responses. J Allergy Clin Immunol. 2015;135(4):1008-18.

19. Zhang C, Li Y, Wu Y, Wang L, Wang X, Du J. Interleukin-6/signal transducer and activator of transcription 3 (STAT3) pathway is essential for macrophage infiltration and myoblast proliferation during muscle regeneration. J Biol Chem. 2013;288:1489-99.

20. Jianru W, Ye T, Kate LEP, Neil C, Gail H, Rowena AB, et al. Tumor necrosis factor $\alpha$ - and interleukin-1 $\beta$-dependent induction of CCL3 expression by nucleus pulposus cells promotes macrophage migration through CCR1. Arthritis \& Rheumatism. 2013;65(3):832-42.

21. Mohamad A, Daniel C, Yusuf AH, Lina MO. Sphingosine-1-phosphate receptor 2. FEBS J. 2013;280:6354-66.

22. Aarthi JJ, Darendelier MA, Pushparaj PN. Dissecting the role of the S1P/S1PR axis in health and disease. J Dental Res. 2011;90:841-54.

23. Nagano T, Edamatsu H, Kobayashi K, Takenaka N, Yamamoto M, Sasaki N, et al. Phospholipase $c \varepsilon$, an effector of ras and rap small GTPases, is required for airway inflammatory response in a mouse model of bronchial asthma. PLoS One. 2014;9(9):e108373. doi:10.1371/journal.pone.0108373.

24. Loh KC, Leong WI, Carlson ME, Oskouian B, Kumar A, Fyrst H, et al. Sphingosine-1-phosphate enhances satellite cell activation in dystrophic muscles through a S1PR2/STAT3 signaling pathway. PLoS One. 2012;7:e37218.

25. Liou CJ, Cheng PY, Huang WC, Chan CC, Chen MC, et al. Oral lovastatin attenuates airway inflammation and mucus secretion in ovalbumin-induced murine model of asthma. Allergy, Asthma Immunol Res. 2014;6(6):548-57.

26. Yan $\mathrm{GH}$, Choi $\mathrm{YH}$. Salidroside attenuates allergic airway inflammation through negative regulation of nuclear factor-kappa B and p38 mitogenactivated protein kinase. J Pharmacol Sci. 2014;126(2):126-35.

27. Shin IS, Park JW, Shin NR, Jeon CM, Kwon OK, Kim JS, et al. Melatonin reduces airway inflammation in ovalbumin-induced asthma. Immunobiology. 2014;219(12):901-8.

28. Mouse Genome Sequencing Consortium, Waterston RH, Lindblad-Toh K, Birney E, Rogers J, et al. Initial sequencing and comparative analysis of the mouse genome. Nature. 2002;420(6915):520-62.

29. Silva DG, Schonbach C, Brusic V, Socha LA, Nagashima T, Petrovsky N. Identification of "pathologs" (disease-related genes) from the RIKEN mouse cDNA dataset using human curation plus FACTS, a new biological information extraction system. BMC Genomics. 2004;5(1):28.

30. Karolien B, Sandra V, Rosette VDH, Hilda W, Inge N, Greet S. The allergic cascade: Review of the most important molecules in the asthmatic lung Immunol Lett. 2007;113:6-18.

31. Gaga M, Ong YE, Benyahia F, Aizen M, Barkans J, Kay AB. Skin reactivity and local cell recruitment in human atopic and nonatopic subjects by CCL2/ MCP-1 and CCL3/MIP-1a. Allergy. 2008;63:703-11.

32. Elena G, Pia J, Clifton FH, Andrew HL, David WHR, Richard JM, et al. Corticosteroid resistant asthma is associated with classical anti-microbial activation of airway macrophages. J Allergy Clin Immunol. 2008;122(3):550-9.

33. Toda M, Kuo CH, Borman SK, Richardson RM, Inoko A, Inagaki M, et al. Evidence that formation of vimentin-mitogen-activated protein kinase (MAPK) complex mediates mast cell activation following FCeRI/CC chemokine receptor 1 cross-talk. J Biol Chem. 2012;287(29):24516-24.
34. Miyazaki D, Nakamura T, Toda M, Cheung-Chau KW, Richardson RM, Ono SJ, et al. Macrophage inflammatory protein-1a as a costimulatory signal for mast cell-mediated hypersensitivity reactions. J Clin Invest. 2005;115:434-42.

35. Toda M, Dawson M, Nakamura T, Munro PM, Richardson RM, Bailly M, et al. Impact of engagement of FCERI and CC chemokine receptor 1 on mast cell activation and motility. J Biol Chem. 2004;279:48443-8.

36. Fifadara NH, Aye CC, Raghuwanshi SK, Richardson RM, Ono SJ. CCR1 expression and signal transduction by murine BMMC results in secretion of TNF-a, TGFß-1, and IL-6. Int Immunol. 2009;21:991-1001.

37. Laffargue M, Calvez R, Finan P, Trifilieff A, Barbier M, Altruda F, et al. Phosphoinositide 3-kinasey is an essential amplifier of mast cell function. Immunity. 2012;16:441-51.

38. Aye CC, Toda M, Morohoshi K, Ono SJ. Identification of genes and proteins specifically regulated by costimulation of mast cell FceRl receotirl and chemokine receptor1. Exp Mol Pathol. 2012;92:267-74.

39. Wang J, Tian Y, Phillips KL, Chiverton N, Haddock G, Bunning RA, et al. Tumor necrosis factor $\alpha$ - and interleukin-1 $\beta$-dependent induction of CCL3 expression by nucleus pulposus cells promotes macrophage migration through CCR1. Arthritis Rheum. 2013;65(3):832-42.

40. Puneet SJ, Meryem B, Ana O, Claudia GE, Richard LP, Juan R, et al. Transactivation of sphingosine-1-phosphate receptors by FceRI triggering is required for normal mast cell degranulation and chemotaxis. J Exp Med. 2004;199(7):959-70.

41. Wang XQ, Mao LJ, Fang QH, Kobayashi T, Kim HJ, Sugiura H, et al. Sphingosylphosphorylcholine induces a-smooth muscle actin expression in human lung fibroblasts and fibroblast-mediated gel contraction via S1P2 receptor and Rho/Rho-kinase pathway. Prostaglandins Other Lipid Mediat. 2014;108:23-30.

42. William SS, Bruce RP. Bryan JM.S1P2 receptor-dependent Rho-kinase activation mediates vasoconstriction in the murine pulmonary circulation induced by sphingosine 1-phosphate. Am J Physiol Lung Cell Mol Physiol. 2010;299(1):L137-45.

43. Du W, Takuwa N, Yoshioka K, Okamoto Y, Gonda K, Sugihara K, et al. S1P2, the $G$ protein-coupled receptor for Sphingosine-1-Phosphate, negatively regulates tumor angiogenesis and tumor growth in vivo in mice. Cancer Res. 2010;70(2):772-81

44. Yapeng G, Tetyana F, Roger $\mathrm{S}$, Jody R. Epithelial cell extrusion requires the sphingosine-1-phosphate receptor 2 pathway. J Cell Biol. 2011;193(4):667-76.

45. Kono M, Belyanseva IA, Skoura A, Frolenkov Gl, Starost MF, Dreier JL, et al. Deafness and stria vascularis defects in S1P2 receptor-null mice. J Biol Chem. 2007;282:10690-6.

46. Kannan RR, Chen ZJ, Singh N, Przekwas A, Delvadia R, Tian G, et al. A Quasi-3D wire approach to model pulmonary airflow in human airways. Int J Numer Method Biomed Eng. 2016. doi:10.1002/cnm.2838.

47. Kannan RR, Guo P, Przekwas A. Particle transport in the human respiratory tract: formulation of a nodal inverse distance weighted Euler-Lagrangian transport and implementation of the Wind-Kessel algorithm for an oral delivery. Int J Numer Methods in Biomed Eng. 2016. doi:10.1002/cnm.2746.

48. Kumar RK, Foster PS. Are mouse models of asthma appropriate for investigating the pathogenesis of airway hyper-responsiveness? Front Physiol. 2012;3(312):1-7

49. Sun Y, Wang J, Li H, Sun L, Wang Y, Han X. The effects of budesonide on angiogenesis in a murine asthma model. Arch Med Sci. 2013;9(2):361-7.

\section{Submit your next manuscript to BioMed Central and we will help you at every step:}

- We accept pre-submission inquiries

- Our selector tool helps you to find the most relevant journal

- We provide round the clock customer support

- Convenient online submission

- Thorough peer review

- Inclusion in PubMed and all major indexing services

- Maximum visibility for your research

Submit your manuscript at www.biomedcentral.com/submit 